\title{
Theory of Mind and Executive Functioning in Alcohol Dependence Syndrome
}

\author{
Preeti Singh ${ }^{1}$, Narendra Nath Samantaray ${ }^{2 *}$, Masroor Jahan ${ }^{3}$
}

\section{ABSTRACT}

Title: Theory of mind and executive functioning in alcohol dependence syndrome. Objectives: The objective of study to examine the relationship of the Theory of Mind (TOM) deficit and Executive Functioning difficulties of the Alcohol Dependence Syndrome (ADS). Methods: Forty-five patients of ADS age range between 18 to 35 years participated in the study. All patients selected for study were inpatients of de-addiction ward of the Ranchi Institute of Neuropsychiatry and Allied Sciences (RINPAS), Ranchi. All participants were tested on measures of TOM tasks and Wisconsin Card Sorting Test (WCST). Results: The result findings suggest that the First Order Deception Tasks (FODT) of TOM was significantly correlated with the WCST's no. of error, percentage of error, non-perseverative error and First Order Deception Tasks (FODT) of Memory with all variables of WCST. Second Order Deception Tasks (FODT) of TOM was significantly correlated with the WCST's percentage of error, non-perseverative error and conceptual level responses. Attribution of Intention Tasks (AIT) of TOM was significantly correlated with the all variables of the WCST. Conclusions: The ADS patients having difficulty in TOM tasks and this difficulty may be related to underlying deficit in executive functioning.

Keywords: Theory of Mind, Executive Functioning, Alcohol Dependence Syndrome.

The Theory of Mind (TOM) is the ability to attribute mental states beliefs, intents, desires, pretending, knowledge or emotions to oneself and others and to understand that others have beliefs, desires, intentions, and perspectives that are different from one's own (Premack and Woodruff, 1978). The TOM term was first used by primatologists and psychologists Premack and Woodruff (1978). The TOM deficits can occur in people with autism spectrum disorders (Baron-Cohen, 1988), schizophrenia (Corcoran and Frith, 2003), attention deficit hyperactivity disorder (Korkmaz, 2011), bipolar disorders (Kerr et al., 2003; Montag et al., 2009), major

\footnotetext{
${ }^{1}$ PhD Scholar, Department of Clinical Psychology, RINPAS, Ranchi, India

${ }^{2}$ Clinical Psychologist, Dept of Clinical Psychology, Mental Health Institute (COE), SCB Medical College \& Hospital, Cuttack, India

${ }^{3}$ Additional Professor, Department of Clinical Psychology, RINPAS, Ranchi, India

*Responding Author

(C) 2016 I P Singh, N Samantaray, M Jahan; licensee IJIP. This is an Open Access Research distributed under the terms of the Creative Commons Attribution License (http://creativecommons.org/licenses/by/2.0), which permits unrestricted use, distribution, and reproduction in any Medium, provided the original work is properly cited.
} 


\section{Theory of Mind and Executive Functioning in Alcohol Dependence Syndrome}

depression disorders, specific language impairments and as well as alcoholics who have suffered brain damage due to alcohol's neurotoxicity, particularly the prefrontal cortex region of the brain (Uekermann, Daum, 2008).

There are several theories which described the different theoretical perspective of the structure and process of the TOM. Some theorists argue that a distinct theory of mind skill need not be invoked at all, but rather that mental state attribution can be completely and more parsimoniously accounted for by general cognitive mechanisms, such as executive functions. These theories support different claims about the existence of specific neural structures that might sub serve theory of mind. There are four major explanation of the TOM by different theories:

The first one is a Modular Theory of TOM claims that theory of mind exists as a distinct, cognitive ability that is functionally dissociable from other cognitive functions. In addition, many modular theorists assert that the theory of mind module is innate, follows a pre-set developmental course, and matures relatively independently from other cognitive skills (BaronCohen, 1995; Leslie \& Roth, 1993). This domain- specific cognitive module has emerged from the study of autism (Baron-Cohen, Leslie, \& Frith, 1985; Leslie \& Thaiss, 1992).

The second one is a Simulation Theory of TOM proposed that theory of mind ability, or attributing mental states to others, can be most accurately conceived of as an act of role-taking (Langdon \& Coltheart, 2001). From this perspective, individuals' interpretations of the world are not guided by a general theory about how minds work, nor do individuals make inferences about the mental states of others, and thereby predict actions. Instead, individuals simulate what reality would look like to another person by mentally placing themselves into that person's perspective, and then predicting what they themselves would do in the other person's place. Simulation theory does not differentiate between abstract, cognitive perspective-taking and concrete, visual perspective-taking that involves mental manipulation of a physical environment. This is in opposition to modular theories, which clearly differentiate mental state inferences from inferences about the concrete, physical world. In addition, simulation theory does not require the meta-representational computations about reality that are imposed by some modular theorists (Leslie \& Roth, 1993).

The third one is a Developing Theory of TOM viewing the theory of mind as a developing, evolving theory about other minds that is revised with experience over time. This theoretical stance posits several different theories of mind that replace one another as an individual's appreciation of alternate, cognitive perspectives becomes increasingly more sophisticated (Gopnik \& Wellman, 1994).

The last but not least one is Executive Function Theory of TOM believes that executive functions are sufficient to perform the mental inference skills attributed to theory of mind, without the invocation of any specialized cognitive skill (Ozonoff et al., 1991). These theorist 
that reject a theory of mind construct argue that the tasks traditionally used to assess theory of mind ability primarily test executive function component skills such as set-shifting and response inhibition. For example, an individual might fail to inhibit a response based upon his own beliefs, and so fail to display his actually intact appreciation of an alternative perspective held by another person. These cognitive- general theorists further claims that the core, meta-representation ability attributed to theory of mind by modularity theorists is merely one example of the general executive capacity for using embedded rules (Frye, Zelazno, \& Palfai,1995). These theorists have also presented evidence that executive function performance predicts theory of mind performance, while theory of mind performance does not predict executive function performance. This suggests that theory of mind is dependent on executive functions while executive functions are not dependent on any theory of mind skill (Frye et al., 1995; Hughes, 1998; Ozonoff et al., 1991). This executive functioning explanation of TOM is primarily focused in this present study.

There are several studies done on the TOM in subjects with alcohol use disorders (Bosco et al. 2013; Maurage et al. 2015; Uekermann et al. 2007; Gizewski et al. 2013) and found that the TOM is impaired in alcohol use disorders (AUD). The impairments observed in alcohol use disorders were related in particular to TOM and executive functions especially working memory (Uekermann et al. 2007). These studies only discussed about the deficit of the TOM and executive functioning in AUD but there were no description was given about the relationship of the executive functioning and TOM variables found in AUD patients. Therefore this study was planned to find out the deficit and interrelationship of different features of TOM tasks and executive functioning in the alcohol dependence disorders (ADS).

\section{METHOD}

\section{Sample:}

The 45 male patients of Alcohol Dependence Syndrome were selected for the study from inpatients of De-addiction ward of Ranchi Institute of Neuro-Psychiatry and Allied Sciences (RINPAS), Kanke, Ranchi. The age range of patients was between 18-35 years and they are educated upto $10^{\text {th }}$ standard. The patient is having more than five years history of substance use, other co-morbid substance abuse history, other co-morbid psychiatric disorders, mental retardation and organic history were excluded.

\section{Tools:}

For the present study following tools were used:

1. Socio-demographic and Clinical Data Sheet: It was semi structured Performa especially designed for this study. It contains information about socio-demographic variables like age, sex, education, marital status, residence, occupation, religion and clinical variables like age of onset of illness, mode of onset of illness, course of illness, duration of illness, medication, history of alcohol or substance abuse, family history of mental illness and co-morbid psychiatric illness. 
2. Theory of Mind Tasks: The three major tasks have been used to assess the Theory of Mind (TOM) in the present study are:

a) False Belief Task (FBT):- A false-belief story intends to test subject's ability to infer false belief and to correctly predict that others can act on the basis of beliefs that misrepresented reality. Tasks involving acknowledgement of a story character's false belief about the world is first order TOM task. Second order false belief tasks assesses the mistaken belief of what one story character thinks about another story character's thought. The task was acquired from the stories developed by Wimmer and Parner (1983), Baron-Cohen, Leslie \& U Frith (1986). These tasks were translated into Hindi as standard procedure with some minor changes in a few words due to their culture specificity (e.g. candy was replaced with chocolate).

b) Deception Task (DT):- Deception entails manipulation of another person's thought making some one believe something false. TOM stories by Frith and Corcoran (1996), showing the mental state and memory questions were used for this purpose. The deception stories involved bluff (first order) and double bluff (second order). These tasks were also translated into Hindi as per standard procedure with some minor changes in a few words due to their cultural specificity.

c) The Attribution of Intention Task (TAIT):- The task consisted of four randomly ordered short comic strips, already designed by Sarfati et al. (1977) for exploring TOM in schizophrenia. Some culture specific changes were done wherever required. Material consisted of a series of 4 randomly ordered short comic strips, represented in a sequence of three pictures sketched in black ink on 39x59 inch card. The sketches were adapted keeping in mind their culture specificity as per the procedure. The content was retained as in the original cards.

The scoring of both false belief as well as deception task was done according to a standardized scoring scheme, which has previously shown good inter-rater agreement (Fletcher et al. 1995; Doody et al., 1998). The scoring of attribution of intention task was done to determine subjects different pattern of selection amongst the cards.

3. Wisconsin Card Sorting Test (WCST) - 64 Card Version: This test was used to assess the executive function of the ADS patients. The 64 card version developed by the Kongs et al. (2000). The normative data were developed of this short version for individuals ranging from $6 \frac{1}{2} 2$ through 89 years of age. The generalizability coefficient of test-retest scores ranged from .39 to .72. The construct and cross-cultured validity was assessed of this short version. The construct validity of the WCST as a measure of problem solving has been examined by comparing the test to other measures by Shute and Huertas (1990). In cross-cultural validity potentially important variables included which is reflects the socio-cultural perspective of the population assessed by the Gasquoine (1999). 


\section{Procedure:}

Initially clinical history and socio-demographic data was collected by the help of case record file and interviewing the patient. Then the TOM tasks were exposed and responses were obtained of the ADS patients diagnosed according to the ICD-10 DCR. Then WCST test was administered on the same patients and data was collected. The obtained data of both tests were scored according to the standard procedures and statistically analysed by using Pearson Product Moment method of correlation coefficient, Mean and Standard Deviation.

\section{RESULT}

\section{Table 1: Correlation between TOM tasks and WCST Variables}

\begin{tabular}{|c|c|c|c|c|c|}
\hline 通号 & 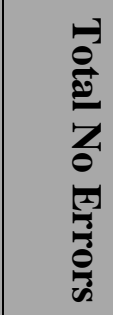 & 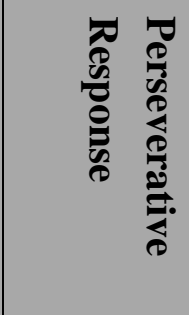 & 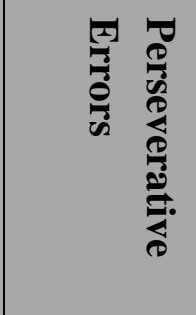 & 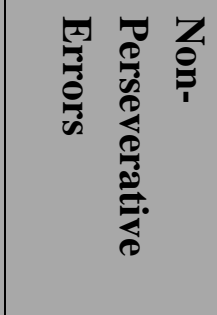 & 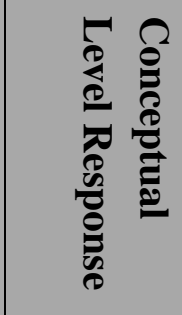 \\
\hline $\begin{array}{l}\text { First Order False Belief } \\
\text { Tasks of TOM }\end{array}$ & -.002 & -.281 & -.311 & -.158 & .000 \\
\hline $\begin{array}{l}\text { First Order False Belief } \\
\text { Tasks of Memory }\end{array}$ & -.002 & -.281 & -.311 & -.158 & .000 \\
\hline $\begin{array}{l}\text { Second Order False Belief } \\
\text { Tasks of TOM }\end{array}$ & -.406 & -.273 & -.306 & -.014 & -.194 \\
\hline $\begin{array}{l}\text { Second Order False Belief } \\
\text { Tasks of Memory }\end{array}$ & -.010 & .059 & .084 & .033 & .176 \\
\hline $\begin{array}{l}\text { First Order Deception } \\
\text { Tasks of TOM }\end{array}$ & .010 & .146 & .112 & .248 & -.276 \\
\hline $\begin{array}{l}\text { First Order Deception } \\
\text { Tasks of Memory }\end{array}$ & .586 & .621 & .620 & .441 & .408 \\
\hline $\begin{array}{l}\text { Second Order Deception } \\
\text { Tasks of TOM }\end{array}$ & -.344 & .122 & .148 & .220 & .329 \\
\hline $\begin{array}{l}\text { Second Order Deception } \\
\text { Tasks of Memory }\end{array}$ & -.376 & -.379 & -.420 & -.286 & -.509 \\
\hline $\begin{array}{l}\text { Attribution of Intention } \\
\text { Tasks }\end{array}$ & .322 & .181 & .180 & .234 & .550 \\
\hline
\end{tabular}

The obtained scores of ADS patients on TOM tasks and WCST variables were correlated and the table 1 findings suggests that the first order deception tasks of TOM were highly correlated with the non-perseverative errors, first order deception tasks of memory highly correlated with the all 
variables of the WCST, second order deception tasks of TOM were highly correlated with the conceptual level responses and attribution of intention tasks were highly correlated with the all WCST variables.

Table 2: Mean and Standard Deviation of TOM and WCST Variables

\begin{tabular}{|l|l|l|}
\hline $\begin{array}{l}\text { TOM/WCST } \\
\text { Variables }\end{array}$ & Mean & Std. Deviation \\
\hline First Order False Belief Tasks of TOM & 1.70 & .48 \\
\hline First Order False Belief Tasks of Memory & 1.70 & .48 \\
\hline Second Order False Belief Tasks of TOM & 1.00 & .67 \\
\hline Second Order False Belief Tasks of Memory & .90 & .74 \\
\hline First Order Deception Tasks of TOM & 1.50 & .53 \\
\hline First Order Deception Tasks of Memory & 1.60 & .52 \\
\hline Second Order Deception Tasks of TOM & 1.10 & .74 \\
\hline Second Order Deception Tasks of Memory & 1.40 & .70 \\
\hline Attribution of Intention Tasks & 2.50 & 1.35 \\
\hline Total No of Errors & 76.30 & 11.09 \\
\hline Perseverative Responses & 79.20 & 15.86 \\
\hline Perseverative Errors & 79.40 & 16.87 \\
\hline Non-Perseverative Errors & 97.60 & 11.91 \\
\hline Conceptual Level Responses & 69.00 & 6.86 \\
\hline
\end{tabular}

The table 2 findings suggest that the highly variable responses obtained in WCST variables of ADS patients except conceptual level of responses. The findings also denotes that the mild impairment in all variables of the WCST except non-perseverative errors was found and moderate impairment in second order false belief tasks of TOM and Memory.

\section{DISCUSSION AND IMPLICATION}

The present study intended to find out the relationship between Theory of Mind and Executive Functioning of Alcohol Dependence Syndrome patients. The result findings suggests that the first order deception tasks of Memory and TOM were highly correlated with the all variables of the WCST reflects that the impairment in manipulation of another person's thought is related to the poor planning ability, anticipation towards changes, poor flexibility and problem in duel task performance. The findings also denotes that the second order deception tasks of TOM highly correlated with the conceptual level responses of the WCST indicates that the impairment to making some one believe something false of TOM is related to the poor logical analysis and forming new concepts of executive functioning. The ability to predict the others future responses on the basis of the earlier incidents were depends on the cognitive flexibility and logical analysis. 
The study findings can be helpful for the mental health professionals to understand the ADS patients' level of deficit in executive functioning and their effect on social perception and interaction. The study results can be useful for the psychotherapeutic formulation and management of these ADS patients. There are several training, research has been done and ongoing for the improvement of the executive functioning of ADS patients which can not only empower the ADS patients cognitive functioning but also their social and individual ability too.

\section{CONCLUSION}

The overall study findings supports the Executive Function Theory of TOM which discussed that the executive functions are sufficient to perform the mental inference skills attributed to theory of mind, without the invocation of any specialized cognitive skill (Hughes et al, 1995; Ozonoff et al., 1991) and the findings also recommend that the mild impairment in executive functioning was related to the moderate impairment in the ability to understand the others intentions, mental states beliefs, desires, pretending, knowledge or emotions and perspectives that are different from one's own.

\section{REFERENCES}

Baron-Cohen, S. (1988). Social and pragmatic deficits in autism: Cognitive or affective?. Journal of autism and developmental disorders, 18(3), 379-402.

Baron-Cohen, S., Campbell, R., Karmiloff-Smith, A., Grant, J., \& Walker, J. (1995). Are children with autism blind to the mentalistic significance of the eyes?. British Journal of Developmental Psychology, 13(4), 379-398.

Baron-Cohen, S., Leslie, A. M., \& Frith, U. (1985). Does the autistic child have a "theory of mind"?. Cognition, 21(1), 37-46.

Baron-Cohen, S., Leslie, A. M., \& Frith, U. (1986). Mechanical, behavioural and intentional understanding of picture stories in autistic children. British Journal of developmental psychology, 4(2), 113-125.

Bertrand-Sarfati, J. (1977). Columnar stromatolites from the early Proterozoic Schmidtsdrift Formation, northern Cape Province, South Africa-Part I: systematic and diagnostic features.

Bosco, F. M., Capozzi, F., Colle, L., Marostica, P., \& Tirassa, M. (2013). Theory of Mind deficit in subjects with alcohol use disorder: An analysis of mindreading processes. Alcohol and Alcoholism, 49(3), 299-307.

Corcoran, R., \& Frith, C. D. (2003). Autobiographical memory and theory of mind: evidence of a relationship in schizophrenia. Psychological medicine,33(05), 897-905.

Doody, G. A., Götz, M., Johnstone, E. C., Frith, C. D., \& Cunningham Owens, D. G. (1998). Theory of mind and psychoses. Psychological medicine, 28(2), 397-405.

Fletcher, P. C., Happe, F., Frith, U., Baker, S. C., Dolan, R. J., Frackowiak, R. S., \& Frith, C. D. (1995). Other minds in the brain: a functional imaging study of "theory of mind" in story comprehension. Cognition, 57(2), 109-128.

Frith, C. D., \& Corcoran, R. (1996). Exploring 'theory of mind'in people with schizophrenia. Psychological medicine, 26(03), 521-530.

Frye, D., Zelazo, P. D., \& Palfai, T. (1995). Theory of mind and rule-based reasoning. Cognitive Development, 10(4), 483-527. 
Gasquoine, P. G. (1999). Variables moderating cultural and ethnic differences in neuropsychological assessment: The case of Hispanic Americans. The Clinical Neuropsychologist, 13(3), 376-383.

Gizewski, E. R., Müller, B. W., Scherbaum, N., Lieb, B., Forsting, M., Wiltfang, J., ... \& Schiffer, B. (2013). The impact of alcohol dependence on social brain function. Addiction biology, 18(1), 109-120.

Gopnik, A., \& Wellman, H. M. (1994). The theory theory. In An earlier version of this chapter was presented at the Society for Research in Child Development Meeting, 1991.. Cambridge University Press.

Hughes, C. (1998). Executive function in preschoolers: Links with theory of mind and verbal ability. The British Journal of Developmental Psychology,16, 233.

Kerr, N., Dunbar, R. I., \& Bentall, R. P. (2003). Theory of mind deficits in bipolar affective disorder. Journal of affective disorders, 73(3), 253-259.

Korkmaz, B. (2011). Theory of mind and neurodevelopmental disorders of childhood. Pediatric research, 69, 101R-108R.

Langdon, R., \& Coltheart, M. (2001). Visual perspective-taking and schizotypy: evidence for a simulation-based account of mentalizing in normal adults. Cognition, 82(1), 1-26.

Leslie, A. M., \& Roth, D. (1993). What autism teaches us about metarepresentation.

Leslie, A. M., \& Thaiss, L. (1992). Domain specificity in conceptual development: Neuropsychological evidence from autism. Cognition, 43(3), 225-251.

Maurage, F., Timary, P., Tecco, J. M., Lechantre, S., \& Samson, D. (2015). Theory of mind difficulties in patients with alcohol dependence: beyond the prefrontal cortex dysfunction hypothesis. Alcoholism: Clinical and Experimental Research, 39(6), 980-988.

Montag, C., Ehrlich, A., Neuhaus, K., Dziobek, I., Heekeren, H. R., Heinz, A., \& Gallinat, J. (2010). Theory of mind impairments in euthymic bipolar patients. Journal of affective disorders, 123(1), 264-269.

Ozonoff, S., Pennington, B. F., \& Rogers, S. J. (1991). Executive function deficits in highfunctioning autistic individuals: Relationship to theory of mind. Journal of child Psychology and Psychiatry, 32(7), 1081-1105.

Premack, D., \& Woodruff, G. (1978). Does the chimpanzee have a theory of mind?. Behavioral and brain sciences, 1(04), 515-526.

Shute, G. E., \& Huertas, V. (1990). Developmental variability in frontal lobe function. Developmental Neuropsychology, 6(1), 1-11.

Uekermann, J., \& Daum, I. (2008). Social cognition in alcoholism: a link to prefrontal cortex dysfunction?. Addiction, 103(5), 726-735.

Uekermann, J., Channon, S., Winkel, K., Schlebusch, P., \& Daum, I. (2007). Theory of mind, humour processing and executive functioning in alcoholism.Addiction, 102(2), 232-240.

Wimmer, H., \& Perner, J. (1983). Beliefs about beliefs: Representation and constraining function of wrong beliefs in young children's understanding of deception. Cognition, 13(1), 103128. 